\section{Tackling AKI: prevention, timing of dialysis and follow-up}

\section{Anitha Vijayan (D)}

Timely diagnosis and dialytic treatment of acute kidney injury (AKI) came to the forefront at the height of the coronavirus disease 2019 pandemic as admissions surged in intensive care units. Research on early diagnosis, timing of initiation of kidney replacement therapy, and appropriate posthospitalization patient care remains essential to tackling the burden of AKI.
Acute kidney injury (AKI) is a major complication in hospitalized patients and is long-term morbidity and mortality, especially in critically ill patients. Timely diagnosis of AKI remains a challenge, as urine output and serum creatinine are suboptimal in detecting early stages of kidney injury. The identification of biomarkers for early diagnosis of AKI remains a work in progress and no single biomarker has been uniformly adopted as an early marker of tubular injury. The TRIBE-AKI consortium evaluated plasma concentrations of pro-and anti-angiogenic markers in 1,444 patients undergoing cardiac surgery, and demonstrated that high post-operative levels of the anti-angiogenic factor vascular endothelial growth factor receptor 1 (VEGFR1) were associated with a high risk of AKI and mortality ${ }^{1}$. By contrast, high levels of the pro-angiogenic factors VEGF and placental growth factor were associated with lower risk of AKI (31\%) and mortality (54\%). Further studies on the role of angiogenesis in the post-injury phase of AKI are needed to delineate whether targeting angiogenesis might expedite kidney recovery and/or improve mortality. A 2020 Acute Disease Quality Initiative (ADQI) consensus statement as [TIMP2 ${ }^{*}[$ IGFBP7] and NGAL, be used to identify populations (mostly patients undergoing cardiac and major abdominal surgery) where interventions to prevent AKI, such as optimizing blood pressure and volume status, or nephrotoxin avoidance, have been shown to improve outcomes ${ }^{2}$. ADQI also recommended that traditional criteria for AKI diagnosis (that is, serum creatinine and urine output) and prognosis (that is, proteinuria) continue to be used in clinical practice, as insufficient evidence supported the routine use of biomarkers to differentiate between associated with substantial short-term and recommended that validated biomarkers, such
AKI and acute kidney disease (AKD; defined as AKI lasting 7-90 days).

Type 2 diabetes mellitus (T2DM) is a major cause of chronic kidney disease (CKD) and kidney failure. Small trials have suggested that T2DM might also be a risk factor for AKI and a large retrospective cohort study conducted by the BEAt-DKD consortium in Scotland further supports its potential as an independent risk factor in the absence of $\mathrm{CKD}^{3}$. In this study, 16,700 participants $(9,417$ with T2DM and 7,283 controls without T2DM) were followed for a median of 8.2 years. Participants without underlying CKD but with T2DM, were five times more likely to develop AKI compared with those without T2DM (121.5 versus 24.6 per 1,000 person-years). Patients with underlying CKD were more than twice as likely to develop AKI when compared with those without CKD. T2DM and other risk factors for AKI, such as malignancy, liver disease or hypertension, must therefore be carefully documented when patients are hospitalized to enable implementation of appropriate AKI prevention strategies.

Critically ill patients with coronavirus disease 2019 (COVID-19) also have a substantial risk of developing AKI, which seems to be characterized by ischaemic tubular injury due to hemodynamic changes caused by a systemic

\section{T2DM and other risk factors} for AKI ... must therefore be carefully documented when

inflammatory response and mechanical ventilation. In one study from New York, USA, $89.7 \%$ of patients on mechanical ventilation developed AKI, compared with $21.7 \%$ of patients who did not require ventilation ${ }^{4}$. Although the aetiology of AKI in these patients is similar to that seen in patients with sepsis or other conditions that lead to severe systemic inflammatory responses, the intense volume of patient admissions overwhelmed hospital capacity to provide timely and routine kidney replacement therapy (KRT). Hospitals rapidly adjusted their practices, for example by implementing hybrid KRT therapies, recruiting non-dialysis personnel, and using non-traditional continuous KRT solutions to ensure delivery of care to every patient who required dialysis ${ }^{5}$.

Preventive strategies such as optimizing volume haemodynamics and volume status, as well as avoiding nephrotoxins, are the mainstay of management of early AKI, but KRT remains the only therapeutic option for severe AKI (FIG. 1). The STARRT-AKI study marked a major development in our understanding of the appropriate timing of initiation of KRT in critically ill patients with $\mathrm{AKI}^{6}$. In this multi-national randomized controlled trial of 3,109 patients, 1,462 patients were randomly assigned to standard strategy and 1,465 to accelerated strategy. In the accelerated strategy group, patients were eligible to start KRT within 12 hours of meeting the criteria of stage 2 AKI. In the standard strategy group, physicians were discouraged from starting KRT unless patients met one of the following criteria: serum potassium $\geq 6.0 \mathrm{mmol} / \mathrm{L}, \mathrm{pH} \leq 7.2$, serum bicarbonate level $\leq 12 \mathrm{mmol} / \mathrm{L}$, severe respiratory failure, or persistence of AKI > 72 hours. The primary end point (90-day all-cause mortality) did not differ between the two groups but the overall patients are hospitalized

\section{Key advances}

- Individuals with type 2 diabetes mellitus (T2DM) are at significantly higher risk of acute kidney injury (AKI) than those without T2DM, independent of underlying chronic kidney disease.

- In patients undergoing cardiac surgery, elevated levels of anti-angiogenic factor vascular endothelial growth factor receptor 1 are associated with a higher risk of AKI and mortality; biomarkers for early diagnosis of AKI remain a work in progress.

- The STARRT-AKI trial demonstrated that early initiation of kidney replacement therapy for patients with AKI in intensive care units did not improve outcomes and was associated with a higher risk of hypotension and hypophosphatemia.

- Albuminuria at 90 days after a hospitalization complicated by AKI is a strong predictor of progressive decline in kidney function. 


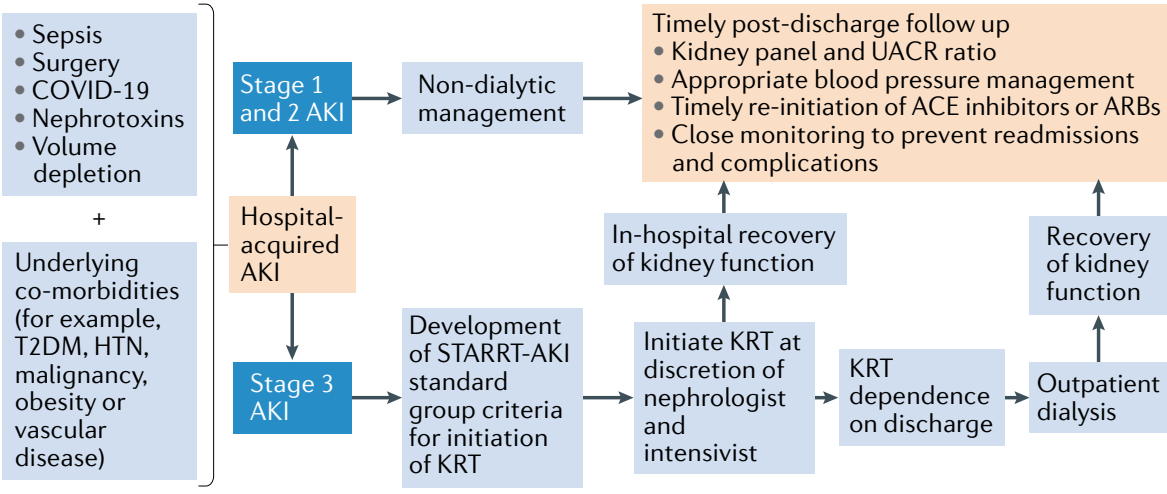

Fig. 1 Approach to the management of patients with acute kidney injury. Risk factors that predispose patients to acute kidney injury (AKI) include sepsis, coronavirus disease 2019 (COVID-19) and exposure to nephrotoxins. In addition, underlying co-morbidities such as type 2 diabetes mellitus (T2DM) and hypertension (HTN) can further increase the risk of AKI. For the majority of patients, AKI will not be severe and kidney replacement therapy (KRT) will not be required, although careful post-hospitalization care remains essential to prevent recurrent AKI. In patients with severe $\mathrm{AKI}$ (stage 3), careful consideration must be given to the timing of KRT to optimize kidney recovery and reduce the need for long-term KRT. ACE, angiotensin-converting enzyme; ARBs, angiotensin II receptor blockers; STARRT-AKI, Standard versus Accelerated Initiation of Renal Replacement Therapy in AKI; UACR, urinary albumin-to-creatinine ratio.

adverse events - specifically hypotension and hypophosphatemia - were higher in the accelerated strategy group. The standard strategy criteria are already used by the majority of nephrologists and this study reaffirms the current approach to KRT initiation in the intensive care unit (ICU). Importantly, twothirds of the patients in this study were in medical ICUs and it might therefore not be possible to completely extrapolate these findings to surgical patients who might need earlier initiation of KRT for volume removal. A post-hoc analysis of this study will investigate whether patients with oliguria might benefit from earlier initiation of KRT.

\section{careful post-hospitalization} care will be essential to prevent a surge in CKD due to COVID-19associated AKI

A considerable proportion of patients with AKI only develop stage 1 or $2 \mathrm{AKI}$ and recover kidney function without needing KRT. The ASSESS-AKI prospective cohort study evaluated the association of post-AKI proteinuria with future deterioration of kidney function, defined as 50\% decrease in estimated glomerular filtration rate (eGFR) or development of kidney failure, in 1,538 hospitalized patients who survived to their baseline study visit 3 months post-hospitalization ${ }^{7}$. The study cohort included 759 patients with or without CKD who experienced in-hospital AKI (defined by a rise in serum creatinine levels of $\geq 50 \%$ or $>0.3 \mathrm{mg} / \mathrm{dl}$ from baseline) and 769 patients who did not have AKI. The two groups were matched for their preadmission CKD status, comorbidities and treatment in the ICU. At the baseline visit, serum creatinine was $1.3 \mathrm{mg} / \mathrm{dl}$ in the AKI group and $1.07 \mathrm{mg} / \mathrm{dl}$ in the control group $(P<0.001)$, and median urinary albumin-to-creatinine ratio (UACR) was $21 \mathrm{mg} / \mathrm{g}$ in the AKI group and $11 \mathrm{mg} / \mathrm{g}$ in those without AKI. Among patients with AKI, 97 (12.6\%) had progression of their kidney disease and a lower post-AKI eGFR was associated with a higher risk for progression of kidney disease (HR 1.5 for each $10 \mathrm{ml} / \mathrm{min} / 1.73 \mathrm{~m}^{2}$ decrease, $95 \%$ CI 1.36-1.66). However, albuminuria was a better predictor of kidney disease progression than eGFR in this study; higher UACR was associated with a higher risk of kidney disease progression (HR 1.53, 95\% CI 1.43-1.64). This report underscores the importance of close follow-up for patients with AKI, as timely intervention in the ambulatory care setting, including appropriate blood pressure management and medication reconciliation, might be key to preventing post-AKI complications. The caring for outpatients after acute kidney injury (COPE-AKI) consortium, which is funded by the US National
Institutes of Health, will evaluate novel interventions versus usual post-hospitalization care for patients with $\mathrm{AKI}^{8,9}$. Crucially, careful post-hospitalization care will be essential to prevent a surge in CKD due to COVID-19-associated $\mathrm{AKI}^{10}$.

In summary, 2020 has demonstrated that AKI continues to be a devastating problem in hospitalized patients, especially in those who are critically ill. Biomarkers for early diagnosis remain a work in progress but recognizing T2DM or COVID-19 as risk factors might facilitate AKI prevention in hospitalized patients. The STARRT-AKI study has provided guidance regarding the timing of initiation of KRT in critically ill patients with AKI, as early initiation of KRT does not seem to benefit most patients and might be associated with harm. Importantly, timely post-hospitalization care has been associated with reduced risk of readmission, recurrent AKI and progression of CKD, which highlights the need to investigate strategies for optimal follow up of patients post-AKI.

Anitha Vijayan iD

Division of Nephrology, Washington University in St. Louis, St. Louis, MO, USA.

e-mail:avijayan@wustl.edu

https://doi.org/10.1038/s41581-020-00390-3

1. Mansour, S. G. et al. The association of angiogenesis markers with acute kidney injury and mortality after cardiac surgery. Am. J. Kidney Dis. 74, 36-46 (2019).

2. Ostermann, M. et al. Recommendations on acute kidney injury biomarkers from the acute disease quality initiative consensus conference: a consensus statement. JAMA Netw Open 3, e2019209 (2020).

3. Hapca, S. et al. The relationship between AKI and CKD in patients with type 2 diabetes: an observational cohort study. J. Am. Soc. Nephrol. https://doi.org 10.1681/ASN.2020030323 (2020).

4. Hirsch, J. S. et al. Acute kidney injury in patients hospitalized with COVID-19. Kidney Int. 98, 209-218 (2020).

5. The Division of Nephrology, Columbia University Vagelos College of Physicians. Disaster Response to the COVID-19 pandemic for patients with kidney disease in New York City. J. Am. Soc. Nephrol. 31, 1371-1379 (2020).

6. STARRT-AKI Investigators et al. Timing of initiation of renal-replacement therapy in acute kidney injury. N Engl J Med 383, 240-251 (2020).

7. Hsu, C. Y. et al. Post-acute kidney injury proteinuria and subsequent kidney disease progression: the assessment, serial evaluation, and subsequent sequelae in acute kidney injury (ASSESS-AKI) Study. JAMA Intern. Med. 180, 402-410 (2020).

8. Siew, E. D. et al. Improving care for patients after hospitalization with AKI. J. Am. Soc. Nephrol. 31, 2237-2241 (2020).

9. US Department of Health and Human Services. Caring for outpatients after acute kidney injury (COPE-AKI). Grants \& Funding https://grants. nih.gov/ grants/guide/rfa-files/rfa-dk-20-012.html (2020)

10. Chan, L. et al. AKI in Hospitalized patients with COVID-19. J. Am. Soc. Nephrol. https://doi.org/ 10.1681/ASN.2020050615 (2020).

Competing interests

A.V. consults for NxStage Inc. and Astute Medical. 